\title{
STICKLER SYNDROME: CORRELATION BETWEEN VITREORETINAL PHENOTYPES AND LINKAGE TO COL $2 A 1$
}

\author{
MARTIN P. SNEAD ${ }^{1.3}$, STEWART J. PAYNE ${ }^{1}$, DAVID E. BARTON ${ }^{1}$, JOHN R. W. YATES ${ }^{2,4}$, \\ LAMYA AL-IMARA ${ }^{5}$, F. MICHAEL POPE ${ }^{5}$ and JOHN D. SCOTT ${ }^{3}$ \\ Cambridge and Harrow
}

\begin{abstract}
SUMMARY
Stickler syndrome is an autosomal dominantly inherited condition characterised by ocular, articular, facial, auditory and oral features. There is locus heterogeneity with about two thirds of families showing linkage to the gene encoding type II procollagen (COL $2 A 1)$. Clinical overlap with Marshall's, Wagner's and other syndromes has caused considerable confusion but the importance of the congenital vitreous anomaly, as first described by Scott, has not previously been emphasised. This study examines the linkage of two vitreo-retinal phenotype subgroups of Stickler syndrome to $C O L 2 A 1$. A total of 97 affected patients from 24 pedigrees were examined. This is the largest published series of Stickler syndrome patients to date and all have undergone full clinical and ophthalmological examination by a single investigator. A clinical classification is proposed based on vitreoretinal phenotype. All patients demonstrating the congenital vitreous anomaly have been designated Stickler syndrome type 1 and those without the congenital vitreous anomaly as Stickler syndrome type 2 patients. There were 69 affected patients from 20 unrelated type 1 pedigrees and 28 affected patients from 4 unrelated type 2 pedigrees. Using two markers at the $C O L 2 A 1$ locus, Stickler syndrome type 1 pedigrees showed complete linkage to $C O L 2 A I$ with a maximum lod score of 12.33 at zero recombination. Linkage to $C O L 2 A 1$ was excluded in the two type 2 pedigrees that were informative. From these data it appears that this clinical classification is a useful first step in resolving the genetic heterogeneity in this condition.
\end{abstract}

In 1965, Stickler et al. ${ }^{1}$ described 11 affected individuals

From: 'Molecular Genetics Laboratory, 'Department of Clinical Genetics and ${ }^{3}$ Vitreo-retinal Unit, Addenbrooke's NHS Trust, Hills Road, Cambridge CB2 2QQ, UK; ${ }^{4}$ Department of Pathology, University of Cambridge, Tennis Court Road, Cambridge CB2 1QP, UK; ${ }^{5} \mathrm{MRC}$ Clinical Research Centre, Watford Road, Harrow, Middlesex HA1 3UJ, UK.

Correspondence to: Martin P. Snead, FRCS, FRCOphth, Vitreoretinal Fellow, Department of Ophthalmology, Box 41, Addenbrooke's NHS Trust, Hills Road, Cambridge CB2 2QQ, UK. in a five-generation pedigree with congenital progressive myopia ranging from 8 to 18 dioptres and arthropathy. Associated features were cleft palate and bilateral, early and total retinal detachment which at that time was considered unamenable to treatment. Two years later Stickler and $\mathrm{Pugh}^{2}$ noted sensorineural deafness in the proband and mother and included photographs which showed a characteristic flattened mid-facial appearance.

The association of articular and vitreous abnormalities in Stickler syndrome led Francomano et al. ${ }^{3}$ to examine, and subsequently establish, linkage to the gene encoding type II procollagen (COL $2 A 1)$ in two pedigrees with a maximum lod score of 3.59 at zero recombination. No clinical data on the patients was included.

The reported clinical similarity and apparent overlap between Stickler's, Marshall's, ${ }^{4}$ Wagner's ${ }^{5.6}$ and Weissenbacher-Zweymuller's syndromes ${ }^{7}$ has caused considerable confusion, and there is controversy as to whether these syndromes are the same ${ }^{8-10}$ or different. ${ }^{11,12}$ COL $2 \mathrm{Al}$ remains implicated in only about two thirds of all cases. ${ }^{13}$ A recent paper ${ }^{14}$ suggesting missense mutations (amino acid substitutions) cause Wagner's syndrome whilst nonsense (premature termination) mutations cause Stickler syndrome does not dissociate the vitreoretinal phenotypes. Frequent retinal detachment and, to a lesser extent, cataract are ascribed to Wagner's syndrome, whereas in fact Wagner's original description ${ }^{5}$ no patient suffered retinal detachment, 'cataracta complicata' was almost universal and myopia was less than 3 dioptres in all cases. Two factors contributing to this confusion are the small size and number of pedigrees available for study, and the failure to discriminate between the characteristic and pathognomonic vitreoretinal phenotypes.

Since type II collagen is the major structural component of secondary vitreous, ${ }^{15}$ which is a largely inert structure, incapable of re-formation, $C O L 2 A l$ mutations might be expected to produce congenital and permanent abnormal- 
ities of the vitreous structure. Attention has been drawn to the congenital vitreous anomaly pathognomonic to a subgroup of these patients by Scott. ${ }^{16}$

This is a study of a series of Stickler syndrome patients all of whom have undergone full clinical and ophthalmological examination including slit lamp biomicroscopy by one of the authors (M.P.S.). Ophthalmological, clinical, radiological, audiometric and echocardiographic features will be described in detail elsewhere. These pedigrees have been classified as those with the congenital vitreous anomaly (type 1) and those without (type 2) and both subsequently tested for linkage to the COL $2 A 1$ gene.

\section{MATERIALS AND METHODS}

Twenty-four Stickler syndrome pedigrees with 105 affected subjects were identified from the database of patients referred to the vitreoretinal service at Addenbrooke's Hospital. All affected and apparently unaffected subjects underwent full clinical and ophthalmological examination by one of the authors (M.P.S.). Informed written consent was received in all cases and prior ethical approval for the study was obtained. The criteria for diagnosis of Stickler syndrome type 1 were as follows:

1. Presence of congenital vitreous anomaly. ${ }^{16}$

and in addition, any three of the following features:

2. Stable congenital myopia with onset prior to 6 years of age.

3. Rhegmatogenous retinal detachment or paravascular pigmented lattice degeneration.

4. Joint laxity with abnormal Beighton score ${ }^{17}$ with or without radiological evidence of joint degeneration.

5. Audiometric confirmation of sensorineural hearing defect.

6. High arched or cleft palate.

The criteria for diagnosis of Stickler syndrome type 2 were as follows.

1. Architecturally abnormal vitreous gel but absence of congenital vitreous anomaly in all affected subjects.

and in addition, any three of the following features:

2. Myopia, stable or progressive, onset at any age.

3. Rhegmatogenous retinal detachment or paravascular pigmented lattice degeneration.

4. Joint laxity with abnormal Beighton score with or without radiological evidence of joint degeneration.

5. Audiometric confirmation of sensorineural hearing defect.

6. High arched or cleft palate.

Sixty-nine affected patients from 20 Stickler type 1 pedigrees and 28 affected patients from 4 Stickler type 2 pedigrees were recruited to the study.

\section{COL 2A1 Polymorphic Markers}

Leukocyte DNA was extracted from 20-30 ml of peripheral blood according to standard procedures.
Hind III Restriction Fragment Length

Polymorphism (RFLP)

Polymerase chain reaction (PCR) to amplify the intron 33 Hind III polymorphism ${ }^{18}$ was carried out in $25 \mu \mathrm{l}$ reaction volumes. Each reaction mix consisted of $400 \mathrm{ng}$ of genomic DNA and $0.12 \mu \mathrm{g}$ of each intron 33 primer (f: 5' AGAGACGAGCGGGCTCAGGA3', r: 5' CCCCCATGGTTTGCTCAGTC3' ), $250 \mu \mathrm{M} 2^{\prime}$-deoxynucleoside $5^{\prime}$-triphosphate mix, $0.63 \mathrm{UT}$ Taq DNA polymerase in a buffer containing $10 \mathrm{mM}$ Tris pH 8.3, $50 \mathrm{mM} \mathrm{KCl}$, $0.01 \%$ gelatin and $2.5 \mathrm{mM} \mathrm{MgCl}_{2}$. The temperature profile was 1.0 minutes each at 94,63 and $73{ }^{\circ} \mathrm{C}$ for 25 cycles.

Digestion of the 581 base pair (bp) PCR product with Hind III yielded two fragments of $428 \mathrm{bp}$ and $153 \mathrm{bp}$ if the polymorphic restriction site was present.

After electrophoresis through a $1.5 \%$ agarose gel at $120 \mathrm{~V}$ for 1.25 hours the alleles were visualised with ethidium bromide staining and ultraviolet illumination.

\section{3' VNTR}

PCR to amplify the COL $2 A 13^{\prime} \mathrm{VNTR}^{19}$ was carried out in $25 \mu$ reaction volumes. Each reaction mix consisted of $400 \mathrm{ng}$ of genomic DNA, $60 \mathrm{ng}$ of each VNTR primer (VNTRf: 5'CAACTGATAAAACAGAGAGC3', r: 5' CTCCTTTGTCATGAACTAGC3' ), $250 \mu \mathrm{M} 2^{\prime}$-deoxynucleoside $5^{\prime}$-triphosphate mix, $0.63 \mathrm{U}$ Taq DNA polymerase in a buffer containing $10 \mathrm{mM}$ Tris pH 8.3, $50 \mathrm{mM}$ $\mathrm{KCl}, 0.01 \%$ gelatin and $2.5 \mathrm{mM} \mathrm{MgCl}_{2}$. In addition, each reaction contained $0.3 \mathrm{ng}$ of forward primer which had been end T4 kinase end labelled with $\left[\gamma^{32} \mathrm{P}\right] \mathrm{ATP}$. The temperature profile was 5 minutes at $95{ }^{\circ} \mathrm{C}$ followed by 35 cycles of $95{ }^{\circ} \mathrm{C}$ for 1.5 minutes, $60{ }^{\circ} \mathrm{C}$ for 1.5 minutes and $72{ }^{\circ} \mathrm{C}$ for 3.0 minutes.

Alleles were separated by electrophoresis in $4 \%$ denaturing polyacrylamide gels run for $6 \mathrm{Wh} / \mathrm{cm}$ and visualised by autoradiography.

\section{Analysis}

Lod scores were calculated using the LIPED computer program. ${ }^{20} \mathrm{~A}$ lod score less than -2.0 was taken as excluding linkage at the corresponding recombination fractions.

\section{RESULTS}

A total of 97 affected patients from 24 pedigrees were examined. There were 69 affected patients from 20 unrelated Stickler type 1 pedigrees and 28 affected patients from 4 unrelated Stickler type 2 pedigrees. Linkage was analysed using two markers at the COL $2 A 1$ locus, the $3^{\prime}$ VNTR $^{19}$ and an intragenic Hind III RFLP. ${ }^{18}$

\section{Stickler Syndrome Type 1}

Ten pedigrees were informative for the Hind III polymorphism, 8 pedigrees were informative for the VNTR and 7 pedigrees were informative for both markers. All informative pedigrees showed linkage to $C O L 2 A 1$. Fig. 1 illustrates a typical type 1 family showing co-segregation of 


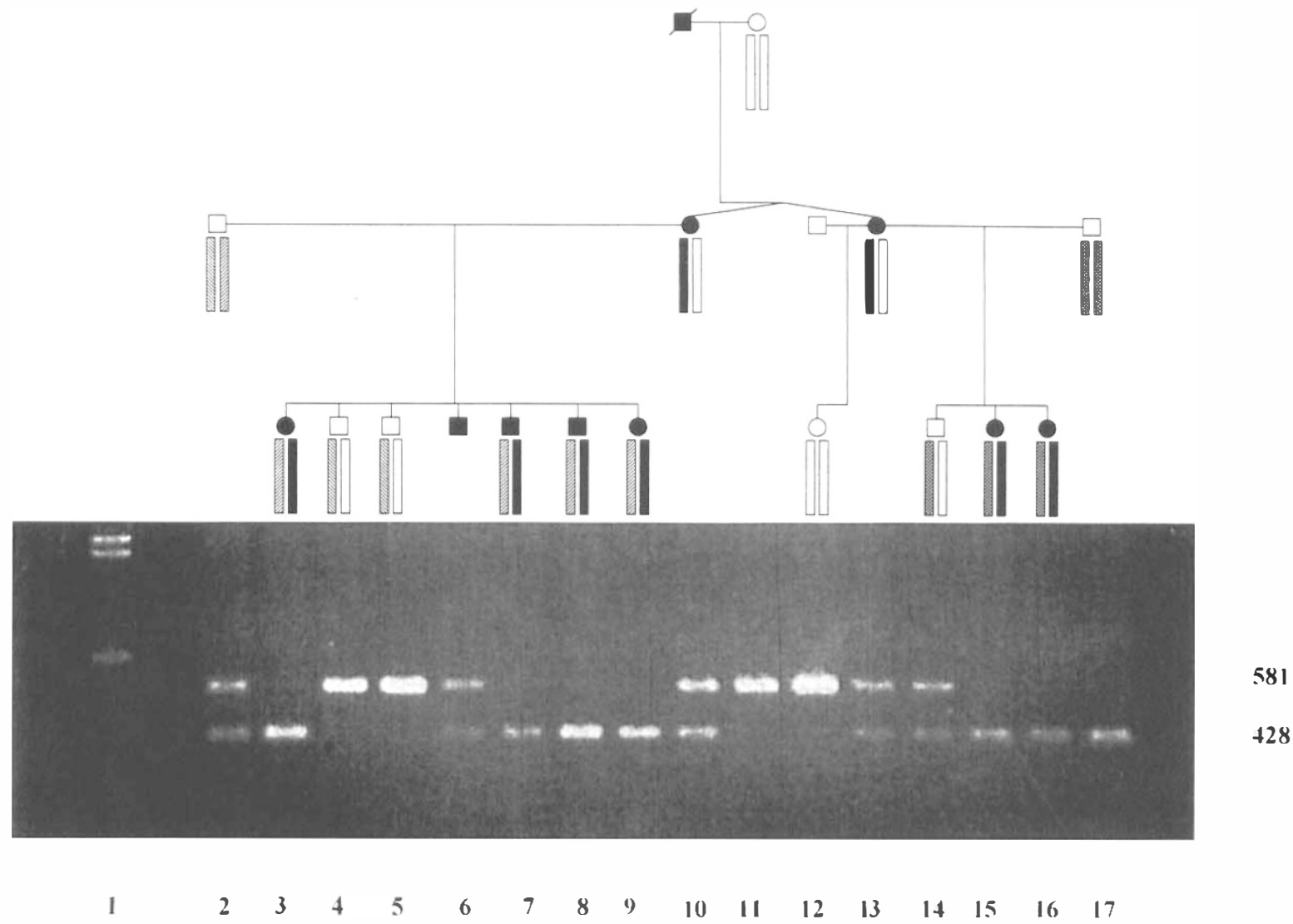

Fig. 1. Pedigree MS 19 (Stickler syndrome type l) show'ing co-segregation of the $428 \mathrm{bp}$ Hind III allele with the disease giving a lod score of $Z \max =3.01, \theta=0$. Note that individual III:4 is uninformative.

the 428 bp Hind III allele with the disease giving a maximum lod score of 3.01 at zero recombination.

The 10 pedigrees that were informative using the Hind III RFLP alone gave a maximum lod score of 8.45 at zero recombination. The 8 pedigrees that were informative for the VNTR alone gave a maximum lod score of 7.93 at zero recombination. Data from both markers were combined to maximise the number of informative meioses but without

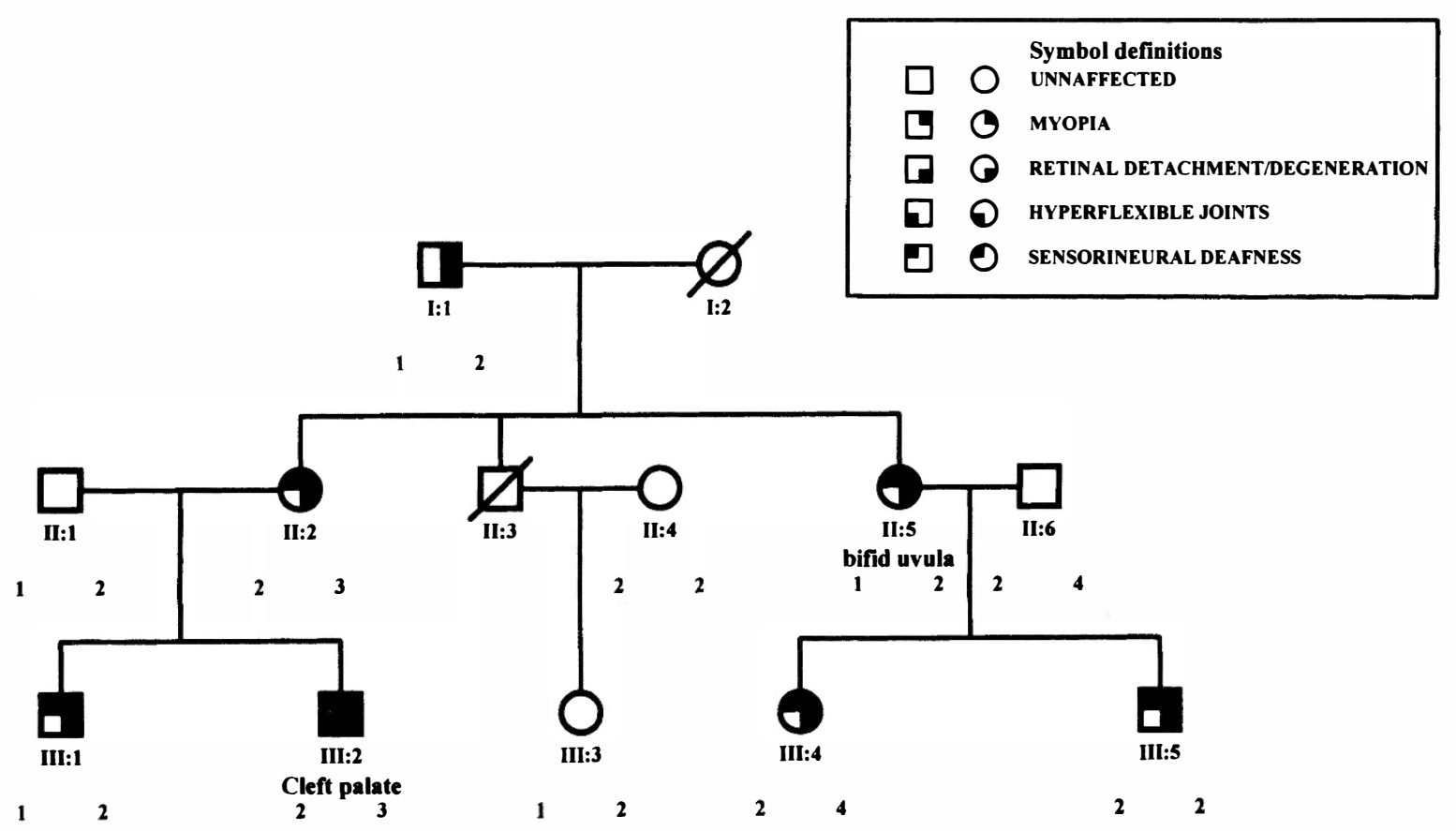

Fig. 2. Pedigree MSI (Stickler syndrome type 2). Numbers represent 3'VNTR alleles; symbol definitions show phenotype. 


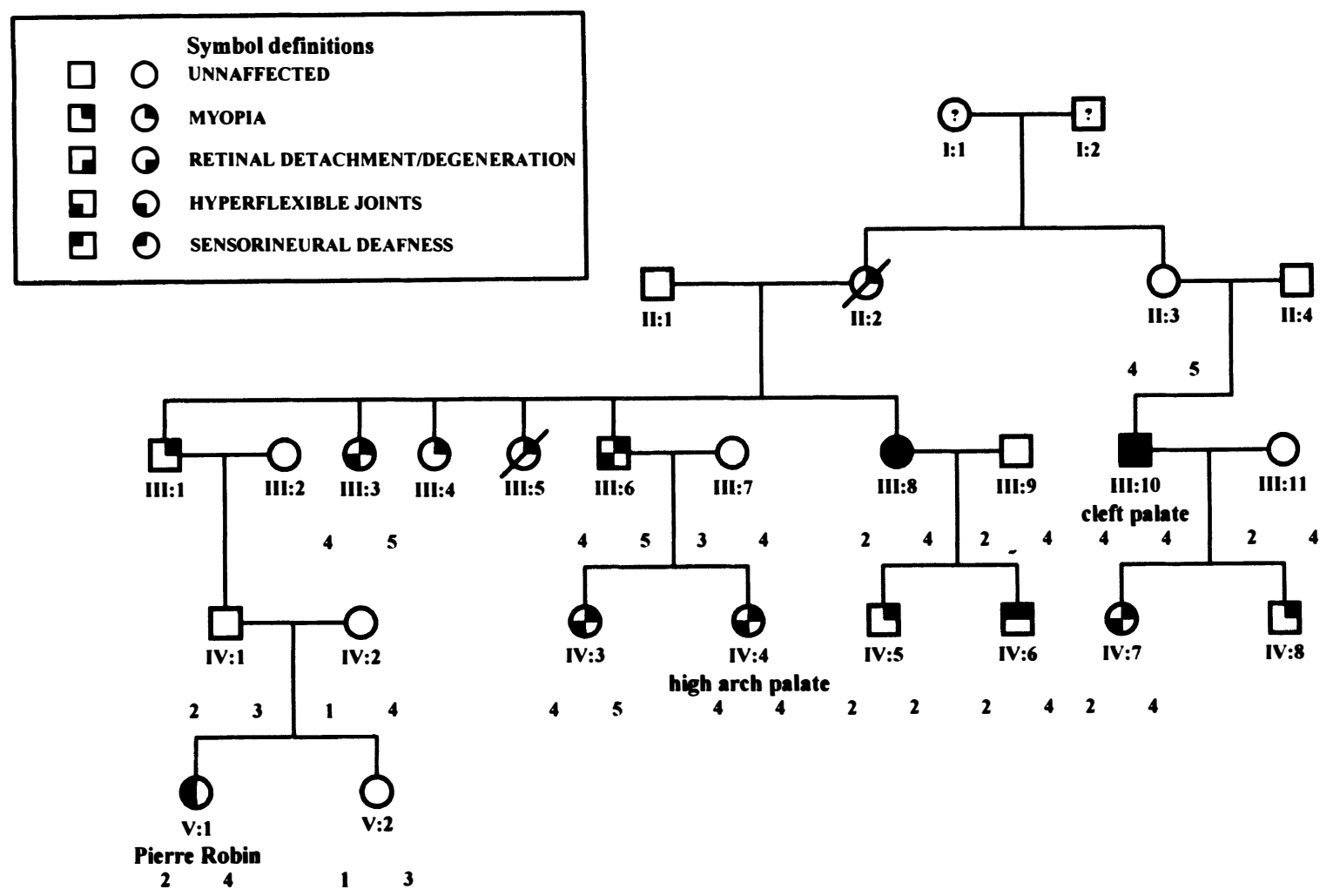

Fig. 3. Pedigree MS 32 (Stickler syndrome type 2). Numbers represent 3'VNTR alleles; symbol definitions show' phenotype.

scoring any twice, and this gave a maximum lod score of 12.33 at zero recombination (Table I).

\section{Stickler Syndrome Type 2}

In 2 pedigrees with Stickler syndrome type 2, linkage to COL $2 A l$ at recombination fraction less than 0.3 was excluded (Table II). These pedigrees are illustrated in Figs. 2 and 3 and demonstrate the phenotypic variation with apparent incomplete penetrance in individuals II:3 and IV:1 in pedigree MS 32. The marker alleles for the $3^{\prime}$ VNTR are given as numbers and each show at least three recombinants between the marker and disease loci. The other two pedigrees were uninformative.

\section{DISCUSSION}

Stickler syndrome has been described as the commonest autosomal dominant connective tissue dysplasia in the North American Midwest. ${ }^{21}$ The ocular complications are severe, including anterior chamber angle anomalies, cataract, and giant retinal tears ${ }^{22}$ which are often bilateral and lead to rhegmatogenous retinal detachment which, untreated, causes blindness. Closed intraocular microsur-

Table I. Two-point lod scores between disease and COL $2 A 1$ markers in Stickler syndrome type 1 families

\begin{tabular}{lrrrrrrr}
\hline & \multicolumn{7}{c}{ Recombination fraction } \\
\cline { 2 - 8 } Marker & \multicolumn{1}{c}{0.0} & 0.001 & 0.05 & 0.1 & 0.2 & 0.3 & 0.4 \\
\hline Hind III & 8.45 & 8.43 & 7.63 & 6.76 & 4.92 & 3.07 & 1.37 \\
3' VNTR & 7.93 & 7.91 & 7.06 & 6.16 & 4.33 & 2.58 & 1.09 \\
Both & 12.33 & 12.31 & 11.10 & 9.86 & 7.17 & 4.46 & 1.99 \\
\hline
\end{tabular}

In combining the data for both markers no meioses were scored twice. gical techniques with vitrectomy and internal tamponade allow successful retinal reattachment but it is likely that prophylactic retinopexy, although not preventing giant retinal tear formation, substantially reduces the risk of progression to retinal detachment and therefore improves visual prognosis.

This paper reports linkage analysis of the largest published series of Stickler syndrome patients to date and all have been examined by a single clinical investigator. There has been considerable uncertainty over the clinical heterogeneity within the Stickler, Marshall, Wagner and other syndromes, ${ }^{8-14}$ but the vitreoretinal differences between the subgroups have been overshadowed by attempts to find or dispel morphological or articular differences.

Vitreous is unique in offering the opportunity to observe, microscopically and non-invasively, the development, anatomical relationships and behaviour of a structure containing predominantly type 2 collagen. Formation abnormalities might be expected if the gene encoding its main structural component were defective. The clinical observation of the congenital vitreous anomaly in a subgroup of Stickler patients with congenital myopia was first described in 1980 by Scott. ${ }^{16}$

Although no biochemical data are available on the vitreous anomaly we can speculate on the consequences of tight linkage between $C O L 2 A l$ and Stickler syndrome pedigrees manifesting the congenital vitreous anomaly. Deletions of entire exons ${ }^{23}$ do not alter the reading frame of mRNA or the ability of shortened procollagen chains to participate in trimer assembly. By random assortment, three quarters of procollagen trimers will consist of het- 
Table II. Two-point lod scores between disease and COL $2 A 1$ markers in Stickler syndrome type 2 families

\begin{tabular}{lccccccccc}
\hline & & \multicolumn{9}{c}{ Recombinant fraction } & & \\
\cline { 3 - 11 } Pedigree & Marker & 0.0 & 0.001 & 0.05 & 0.1 & 0.2 & 0.3 & 0.4 & $\begin{array}{l}\text { Exclusion } \\
\text { of linkage }\end{array}$ \\
\hline MS 1 & $3^{\prime}$ VNTR & $-\infty$ & -7.19 & -2.16 & -1.33 & -0.58 & -0.22 & -0.05 & 0.055 \\
MS 32 & $3^{\prime}$ VNTR & $-\infty$ & -5.32 & -1.51 & -0.74 & -0.12 & -0.07 & -0.07 & 0.03 \\
\hline
\end{tabular}

erotrimers (normal and abnormal chains) and will be unstable and not secreted. The remaining quarter (one eighth each) will be made up of homotrimers (normal or abnormal chains) which might be expected to have normal stability and secretion. This hypothesis is consistent with the clinical appearance: an apparently vestigial vitreous gel occupying the immediate retrolental space, posterior to which there is no discernible collagen gel structure at all. This feature is evident at birth in these patients and remains unchanged throughout life.

In contrast, no Stickler type 2 patient showed the retrolental vitreous anomaly. The type 2 vitreous gel architecture is also congenitally abnormal but with apparently very limited and random fibrils coursing through the entire posterior segment space. This may be associated with areas of localised, or even complete, posterior hyaloid membrane separation.

The present results provide evidence to support at least two distinct phenotypic and genetic subgroups in Stickler syndrome. We have been able clearly to link pedigrees with congenital vitreous anomaly to the COL $2 \mathrm{Al}$ gene and therefore propose a clinical classification, designating this group as Stickler syndrome type 1. Those Stickler syndrome patients with congenitally defective vitreous gel architecture but without vitreous anomaly as described by Scott, have been designated for present purposes as type 2 , and in the pedigrees which were informative, linkage to COL $2 A 1$ has been excluded at recombination fractions up to 0.03 . This region of exclusion would extend to the flanking marker D12S18 distally and well beyond the COL $2 A 1$ gene which encompasses only 30 kilobases. $^{24}$ This clinical classification will help in the search for the Stickler type 2 gene(s) and linkage of these pedigrees to other candiate genes is being examined. Clinical examination of vitreous structure indicates that it is highly likely that the genes encoding the other collagens that associate with, and architecturally stabilise, type 2 collagen will be implicated in some Stickler syndrome type 2 pedigrees. Linkage to other candidate genes is being examined.

If further studies confirm that the congenital vitreous anomaly is consistently associated with mutations in the COL $2 \mathrm{Al}$ gene, this will have useful clinical applications, particularly for carrier detection by genetic linkage or mutation analysis.

The authors are grateful to the many consultants who have referred patients to the vitreoretinal and clinical genetic services at Addenbrooke's Hospital and also allowed access and facilities to examine patients at their departments. M.P.S. is grateful to the Oxford Ophthalmological Congress Research Scholarship scheme and the Addenbrooke's NHS Trust Endowment Fund for support during the laboratory phase of this project.

\section{REFERENCES}

1. Stickler GB, Belau PG, Farrell FJ, Jones JD, Pugh DG, Steinberg AG, Ward LE. Hereditary progressive arthroophthalmopathy. Mayo Clin Proc 1965;40:433-55.

2. Stickler GB, Pugh DG. Hereditary progressive arthro-ophthalmopathy. II. Additional observations on vertebral abnormalities, a hearing defect, and a report of a similar case. Mayo Clin Proc 1967;42:495-500.

3. Francomano CA, Liberfarb RM, Hirose T, Maumenee IH, Sreeten EA, Meyers DA, Pyeritz RE. The Stickler syndrome: evidence of close linkage to the structural gene for type II collagen. Genomics 1987;1:293-6.

4. Marshall D. Ectodermal dysplasia. Am J Ophthalmol 1958;45:143-56.

5. Wagner H. Ein bisher unbekanntes Erbleiden des Auges (Degeneratio hyaloideo-retinalis hereditaria), beobachtet im Kanton Zurich. Klin Monatsbl Augenheilkd 1938;100: 840-57.

6. Bohringer HR von, Dieterle P, Landolt E. Zur klinik und pathologie der Degeneratio hyaloideo-retinalis hereditaria (Wagner). Ophthalmologica 1960;139:330-8.

7. Weissenbacher G, Zweymuller E. Coincidental occurrence of Pierre Robin and foetal chondrodysplasia. Monatsschr Kinderheilkd 1964;112:315-7.

8. Kelly TE, Wells HH, Tuck KB. The Weissenbacher-Zweymuller syndrome: possible neonatal expression of the Stickler syndrome. Am J Med Genet 1982;11:113-9.

9. Winter RM, Baraitser M, Laurence KM, Donnai D, Hall CM. The Weissenbacher-Zweymuller, Stickler, and Marshall syndromes: further evidence for their identity. Am J Med Genet 1983;16:189-99.

10. Baraitser M. Marshall/Stickler syndrome. J Med Genet 1982;19:139-40.

11. Fryer AE, Upadhyaya M, Littler M, Bacon P, Watkins D, Tsipouras P, Harper PS. Exclusion of COL2A1 as a candidate gene in a family with Wagner-Stickler syndrome. J Med Genet 1990;27:91-3.

12. Ayme S, Preus M. The Marshall and Stickler syndromes: objective rejection of lumping. J Med Genet 1984;21:34-8.

13. Vintiner GM, Temple IK, Middleton-Price HR, Baraitser M, Malcolm S. Genetic and clinical heterogeneity of Stickler syndrome. Am J Med Genet 1991;41:44-8.

14. Korkko J,RitvaniemiP, Haataja L, Kaariainen H, Kivirikko KI, Prockop DJ, Ala-Kokko L. Mutation in Type II procollagen (COL2A1) that substitutes aspartate for glycine $\alpha \mathrm{l}-67$ and that causes cataracts and retinal detachment: evidence for molecular heterogeneity in the Wagner syndrome and Stickler syndrome (arthro-ophthalmopathy). Am J Hum Genet 1993;53:55-61.

15. Scott JE. The chemical morphology of vitreous. Eye 1992;6:553-5.

16. Scott JD. Congenital myopia and retinal detachment. Trans Ophthalmol Soc UK 1980;100:69-71.

17. Beighton P. McKusick's heritable disorders of connective tissue. 5th Ed. St Louis: CV Mosby, 1993:199-206.

18. Tsuneyoshi T, Sokolov BP, Prockop DJ. PCR detection of a Hind III polymorphism in the human gene for type II procollagen (COL2A1). Nucleic Acids Res 1991;19:4571.

19. Stoker NG, Cheah KSE, Griffin JR, Pope FM, Solomon E. A highly polymorphic region 3 to the human Type II collagen gene. Nucleic Acids Res 1985;13:4613-22.

20. Ott J. Estimation of the recombination fraction in human 
pedigrees: efficient computation of the likelihood,for human linkage studies. Am J Hum Genet 1974;26:588-97.

21. Herman J, France TD, Spranger JW, Opitz JM, Wiffler C. The Stickler syndrome (hereditary arthro-ophthalmopathy). Birth Defects, Orig Art Serv 1975;2:76-103.

22. Scott JD. Giant tear of the retina. Trans Ophthalmol Soc UK 1975;95:142-4.
23. Lee B, Vissing H, Ramirez F, Rogers D, Rimoin D. Identification of the molecular defect in a family with spondyloepiphyseal dysplasia. Science 1989;244:978-80.

24. Cheah KSE, Stoker NG, Griffin JR, Grosveld FG, Solomon E. Identification and characterisation of the human type II collagen gene (COL2A1). Proc Natl Acad Sci USA 1985;82:2555-9. 